\title{
Mathematics, mathematics education and the mouse
}

\author{
SIU Man Keung \\ Department of Mathematics \\ University of Hong Kong
}

\section{The mouse}

Readers may guess from the title of this article that it has to do with IT (information technology), and they are right. However, they will be disappointed to find that this article does not tell them how to use IT in teaching mathematics, because the author is not qualified to speak on that topic. The emphasis is in another direction, which will become apparent towards the end of this section.

Let me come straight to the mouse. What is nowadays commonly referred to as the mouse made its debut on December 9, 1968. It was invented by Douglas C. Engelbart, a pioneer of human-computer interaction who invented many more things besides, with his team at Stanford Research Institute. When Engelbart received a patent for the wooden shell with two metal wheels - the first mouse - on November 17, 1970, the device was described as an " $X-Y$ position indicator for a display system" [1, Chapter 3].

In a prophetic essay that appeared in 1963, Engelbart already depicts what are nowadays common features on a computer $[2, \mathrm{p} .49]$ :

\footnotetext{
"In this stage, the symbols with which the human represents the concepts he is manipulating can be arranged before his eyes, moved, stored, recalled, operated upon according to extremely complex rules - all in very rapid response to a minimum amount of information supplied by the human, by means of special cooperative technological devices."
}

Technological devices aside, Engelbart announces a key message in his essay, namely, that the computer is not just a tool for efficiency, but a tool which enhances intelligence, and as such it will change our ways of thinking about our world. In [2, p.49] he said, “... we might imagine some relatively straightforward means of increasing our external symbolmanipulation capability and try to picture the consequent changes that could evolve in our language and method of thinking." Take word processing as an example. He outlines the principle of word processing and comments on its influence on the process of writing, far more than just providing a faster typewritter [2, pp.41-42]: 
"This hypothetical writing machine thus permits you to use a new process of composing text. For instance, trial drafts can rapidly be composed from rearranged excerpts of old drafts, together with new words or passages which you insert by hand typing. Your first draft may represent a free outpouring of thoughts in any order, with the inspection of foregoing thoughts continuously stimulating new considerations and ideas to be entered ... You can integrate your new ideas more easily, and thus harness your creativity more continuously, if you can quickly and flexibly change your working record."

The theme of this article is to discuss this kind of influence on the learning and teaching of mathematics.

\section{Current state of the subject (in the eyes of students and the public)}

Recently I came across a book titled Who Needs Classical Music? Cultural Choice and Musical Value by Julian Johnson and read the following passages:

"Music educators occupy a difficult position. Their attempts to introduce students to something that, outside the classroom, they are unlikely to encounter let alone engage with, are resisted not only by students in the classroom but, increasingly, are opposed from outside the classroom, too. A wider academic self-doubt about the claims of classical music has an obvious effect on music education. The new style is eclectic and plural - by celebrating everything, one offends nobody. Thus, the education system reproduces the market place: it presents students with a multiplicity of choices but, all too often, without the deeper analysis of those products that would make choice a meaningful activity." [5, pp.118-119]

"Central to my argument is the idea that classical music is distinguished by a selfconscious attention to its own musical language. Its claim to function as art derives from its peculiar concern with its own materials and their formal patterning, aside from any considerations about its audience or its social use." [5, p.3]

"... that it relates to the immediacy of everyday life but not immediately. That is to say, it takes aspects of our immediate experience and reworks them, reflecting them back in altered form. In this way, it creates for itself a distance from the everyday while preserving a relation to it." $[5, \mathrm{p} .5]$

These passages strike a chord with me, for these same passages ring equally true when the word "mathematics" is substituted for the word "(classical) music" whenever it appears! Mathematics education and music education face a similar plight. It leads one to ask: are we in the mathematical community a group that is too inward-looking? 
Ironic as it may seem, while nobody would deny the importance and applicability of mathematics, the subject has a more and more diminished appeal to the public - diminished motivation, diminished enthusiasm, diminished seriousness, and hence diminished enrollment of maths majors. Why is it like that? There are external factors, some of which are not within the control of the mathematical community. But there are internal factors which the mathematical community may do well to reflect upon. In this article I will focus on one of these internal factors having to do with IT. (I should perhaps point out at the beginning that by a factor I do not always attach to it a negative label, just that I like to reflect upon it.)

\title{
3. What some of our students are like
}

Let me get right down to teaching mathematics in the classroom. Many teachers may share the kind of frustration so vividly depicted in [9, p.7] (at least I do):

\begin{abstract}
"Have you ever encountered students who make extraordinary errors in the midst of supposedly routine calculations?

Have you ever encountered students who seem not to remember what they were taught last year, last term, or even last week?

Have you ever encountered students who score well in tests on routine tasks but do not seem to think of using those techniques in other contexts?

Have you ever encountered students who seem to understand (they pass tests) but who complain about not understanding?"
\end{abstract}

I will cite two anecdotes from my own classroom experience.

(1) I once set a rather standard examination question in a calculus class: The plane $x+y=1$ intersect the surface $z=x y$ in a curve. Locate the highest and lowest points (measured from the Oxy-plane) on this curve, if any.

It can be solved as an extremum problem with constraint or even as an extremum problem in one-variable calculus after reduction. (In fact, knowledge on quadratic form in school mathematics already suffices.) However, it turned out I got many different "solutions" besides the correct one.

"Solution 1": $z=x y$ and $x+y=1$. Hence $x y-z=x+y-1$, so $x y-z-x-y+1=0$. Set $F(x, y, z)=x y-z-x-y+1 . \frac{\partial F}{\partial x}=y-1=0, \frac{\partial F}{\partial y}=x-1=0$, $\frac{\partial F}{\partial z}=-1=0$. The result is impossible, therefore there is no extremum point.

"Solution 2": $z=x y$ and $x+y=1$. Hence $x y-z=x+y-1$, so $z=x y-x-y+1$. $\frac{\partial z}{\partial x}=y-1=0, \frac{\partial z}{\partial y}=x-1=0$, Hence $(1,1)$ is a critical point. $\left(\frac{\partial^{2} z}{\partial x \partial y}\right)^{2}-$ $\left(\frac{\partial^{2} z}{\partial x^{2}}\right)\left(\frac{\partial^{2} z}{\partial y^{2}}\right)=1^{2}-0=1>0$. We conclude that $(1,1)$ is a saddle point. 
"Solution 3": $z=x y$ and $x+y=1$. Hence $(x+y)^{2}=1$, so $x^{2}+y^{2}+2 x y=1$, or $x^{2}+y^{2}+2 z=1$, or $z=\frac{1}{2}\left(1-x^{2}-y^{2}\right) \cdot \frac{\partial z}{\partial x}=-x=0, \frac{\partial z}{\partial y}=-y=0$. Hence $(0,0)$ is a critical point. Since $\left(\frac{\partial^{2} z}{\partial x \partial y}\right)^{2}-\left(\frac{\partial^{2} z}{\partial x^{2}}\right)\left(\frac{\partial^{2} z}{\partial y^{2}}\right)=0^{2}-(-1)(-1)=-1<0$ and $\frac{\partial^{2} z}{\partial x^{2}}=-1<0$, We conclude that $(0,0)$ is a local maximum point.

"Solution 4": $z=x y$ and $x+y=1$. Hence $z / x=y=1-x$, so $z=x-x^{2}$ or $z-x+x^{2}=0$. Set $F(x, z)=z-x+x^{2} \cdot \frac{\partial F}{\partial x}=-1+2 x=0, \frac{\partial F}{\partial z}=1=0$. The final result is impossible, therefore there is no extremum point.

If the student had looked at a geometric picture, the solution would have become quite clear, and so was the mistake occurring in each "solution". However, some students are too much interested in just learning a 'recipe' that they unknowingly put on blinkers [14].

(2) In a class in abstract algebra I set a rather standard homework problem: Show that the function $F: \mathbb{Z}[X] \rightarrow \mathbb{Z}[\sqrt{2}]$ defined by $F(f(X))=f(\sqrt{2})$ is a ring homomorphism which is surjective but not injective.

A student came to say that he had difficulty with the problem. He could give me the correct definitions of a ring homomorphism, a monomorphism, an epimorphism, but I could see that he was reciting the definitions without really knowing what these notions were about. When I asked him what his difficulty was, he mumbled, "When I read the textbook on that section on homomorphism, there are two elements $x_{1}, x_{2}$ involved; you know, $f\left(x_{1}+x_{2}\right)=f\left(x_{1}\right)+f\left(x_{2}\right), f\left(x_{1} x_{2}\right)=f\left(x_{1}\right) f\left(x_{2}\right), \cdots$. You work with the $x_{1}, x_{2}, \ldots$ and it gets somewhat complicated $\cdots$. Let me take $x_{1}, x_{2}$ in $\mathbb{Z}[X]$, then $f\left(x_{1}+x_{2}\right)=$ $f\left(x_{1}\right)+f\left(x_{2}\right)$; hey, isn't that something like what I learnt in linear algebra? But now I should look at $F\left(f\left(x_{2}+x_{2}\right)\right)=\cdots$; how can I get the $f(\sqrt{2})$ in ? I cannot get the $f(\sqrt{2})$ in!' When I pointed out to him that his $x_{1}, x_{2}$ were polynomials in the indeterminate $X$ with integral coefficients and asked him what he meant by $f\left(x_{1}+x_{2}\right)$ and indeed what his $f$ was, he looked totally perplexed.

It seems strange that it does not occur to many students that the first step is to make clear what the objects under investigation are. Rather, they would try hard to seize on something which looks familiar, something they have learnt before. Without thinking they would simply lean on this something to cope with the situation they are facing. In itself, this way of making a connection between what has been learnt and what is newly learnt is not a bad idea at all - indeed it is a good practice. However, the cavalier attitude and the haste in making the connection without thinking are in question.

Frequently it is not the content proper which baffles our students - at least not yet. Many of them are baffled even before coming to the content proper! They are not prepared to sit down calmly to face the situation in a sensible way. Sitting down calmly to face the situation in a sensible way does not guarantee that one can resolve the problem, but at 
least one knows what one is facing and where the difficulty lies. Otherwise, one will end up with a hit-and-miss mentality (later in this article I will call this a "mouse-clicking" mentality). Worse yet, if the student somehow hits without misses (but does not have a clue why he hits), then he would mistakenly think that he knows it but in fact he does not. One day this piece of muddled knowledge will come up to haunt him as a learning obstacle.

\section{On learning mathematics}

In [13] Henri Poincaré said, "No doubt it is hard for a teacher to teach what does not satisfy him entirely, but the satisfaction of the teacher is not the sole object of teaching. We have first to concern ourselves with the pupil's state of mind, and what we want it to become." It is important to try to understand how our students learn, even though not everybody learns in the same manner. In [11, Chapter 5] Seymour Papert coins the word "mathetic" for the art of learning as an analogue to "pedagogy" for the art of teaching. The very word "mathematics" has its Greek etymology of "that which is to be learnt", so mathematics is something to be learnt, not just something being taught.

How do this generation of students learn? A special double issue of Newsweek (August 25, 2003 - September 1, 2003) carried on the cover the headline "Bionic Kids: How Technology Is Altering the Next Generation of Humans". One of the articles bears the title "Log on and learn", in which two points merit attention:

"Children's brains are growing adept at handling a variety of visual information."

"Kids are getting better at paying attention to several things at once. But there is a cost, in that you don't go into any one thing in much depth."

The IT age breeds a generation with a different working habit and a different learning habit, even a different mentality, from that of the older generation, i.e. their parents and teachers. The young generation can react much faster to stimuli and can cope with multitasking with much more ease than their parents and teachers. On the other hand, they may lack the patience and the degree of concentration like their parents and teachers, and they are less inclined to work on a single task in as much depth. There is no dearth of books on both the positive aspect (such as $[10,12])$ and the negative aspect (such as $[16$, 17]). It does well to listen to both sides.

If I ask students whether a certain given quantity A is smaller than, or equal to, or larger than some other given quantity B, many would immediately activate their mental 'mouses'. A dialogue may go on as follows:

"Smaller?"

"No." 
"Larger?"

"No."

"Equal." (Bingo!)

Instead of giving a "yes/no" answer I would respond with "why?" to prompt the student to think. Alas, many of them do not like to think. In their learning environment they click the mouse to get a quick "yes/no" answer so that they learn by trial and error. A wrong answer would cost them practically nothing, while the time spent in figuring out a reasonable answer is much more than clicking the mouse to find out if the answer is correct or wrong. No wonder not many students nowadays will be sufficiently patient to unravel the meaning in the statement of a problem of the following sort: $A$ affirms that $B$ denies that $C$ declares that $D$ is a liar. Knowing that $A, B, C, D$ each speaks the truth once in three times (indpendently), figure out the probability that $D$ was telling the truth? (This problem was posed in 1950 by the British astronomer - mathematician Sir Arthur Stanley Eddington in volume 57 of American Mathematical Monthly, and was explained in [3].) There is no mental 'mouse' to click! Some students, having been attracted to the problem for its playful overtone, may still harbour a sustained interest to wrestle with the problem. But what about a more standard-looking problem in a mathematical textbook, which demands a similar task of unravelling the meaning in the statement: Let $f: \mathbb{R} \rightarrow \mathbb{R}$ be continuous and $S$ be a subset of $\mathbb{R}$ such that every sequence in $S$ has a convergent subsequence whose limit belongs to $S$. Show that for each $\varepsilon>0$, there is a $\delta>0$ such that $x, x^{\prime} \in S$ and $\left|x-x^{\prime}\right|<\delta$ imply $\left|f(x)-f\left(x^{\prime}\right)\right|<\varepsilon$ ? (A related debatable issue is whether such type of problems merits as much attention today as in the past.)

We are still inclined to advise students in our classes to possess a calm mind and to exert concentrated effort in facing a complex situation in the study of mathematics. However, in some other subjects the "mouse-clicking" technique may have become the norm and even more effective. In some cases the total time spent in trying out all alternatives and seeing which one works may be much less than the time spent in reasoning in advance which alternative to select. In some cases the visual display may inform us more than what we can obtain from deductive argument in an abstract context. Having been brought up in such a culture, would students be convinced otherwise? We should let students realize for themselves why in mathematics the "mouse-clicking" technique does not work, mainly because in most cases there does not exist an "overlord" to decide for them whether the selection is correct or not.

Thinking in depth is a time-honoured virtue to be maintained in this IT age. Our ancestors already explained clearly this point in one of the oldest extant mathematical text in Chinese, Zhoubi Suanjing (The Arithmetical Classic of the Gnomon and the Circular Paths) which is believed to be compiled in between the 5 th and the 2 nd century B.C. It is expressed in the form of a dialogue between Rongfang $(\mathrm{R})$ and Chenzi $(\mathrm{C})$ : 
R: $\cdots$ can a fellow as stupid like me learn this way of mathematics?

C: Of course you can. What you have learnt in elementary arithmetic is sufficient to let you go on to learn it. But you must be willing to think continually in earnest.

(A few days later, Rongfang came back to Chenzi.)

R: I cannot figure it out. May I enquire again?

C: This is because you have thought about it but not yet to the point of maturity $\cdots$. You cannot yet generalize what you have learnt $\cdots$. The mathematics is simple to explain but has wide applications. After understanding one category of problems one can infer the reasoning for a variety of other categories. ‥ What makes it difficult to be well versed in the way of mathematics is that when one has learnt it one worries about a lack of breadth; when one has attained breadth, one worries about a lack of practice; when one has attained practice, one worries about a lack of ability to understand. To be able to compare and contrast different categories of problems, that is the mark of an intelligent person.

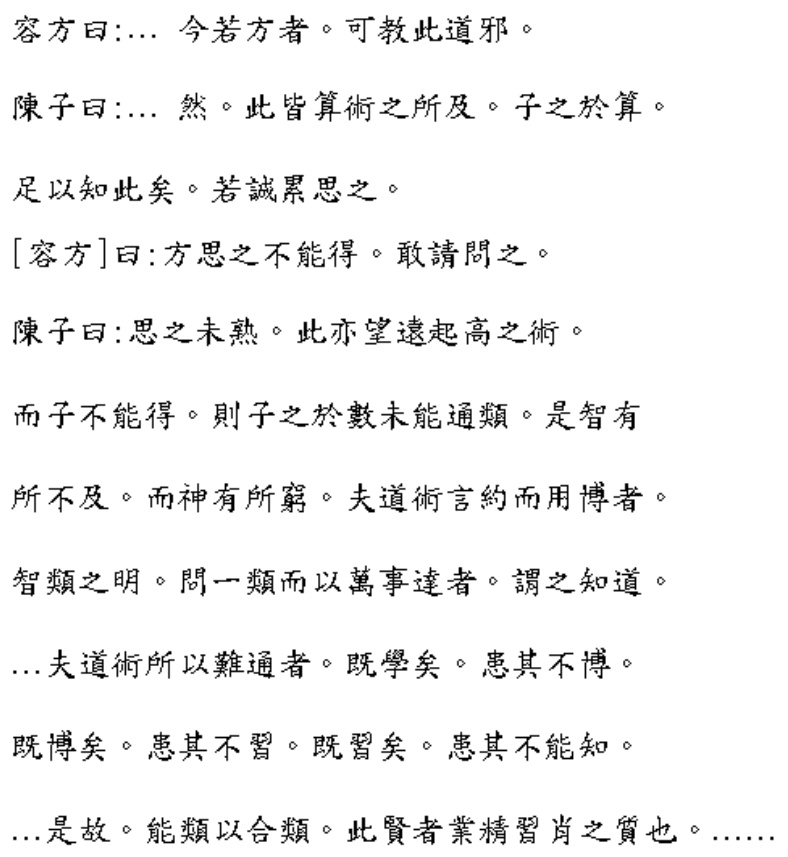

\section{Three examples touching on content}

The advance in computing power may bring along a shift in attention to topics taught in the classroom or a variation in the way they are taught. Certain topics to which we paid much attention in the past may not require as much attention today, or can be taught with a different perspective. Although I express a general 'disclaimer' to my qualification in discussing the integration of IT in the teaching of mathematics, I do admit that it has its 
role to play. A more in-depth discussion on this issue will be firstly, beyond my expertise and secondly, has to be subject-specific. In this article let me just give three examples to illustrate some points of interest.

(1) Back in the 1980s I made use of a programmable calculator in calculus classes to illustrate the idea of approximating a function by polynomial functions, i.e. the Taylor series expansion of a given function. Today with the computer the effect can be made more dramatic and the feature on convergence can be made more lucid (see Figure 1).
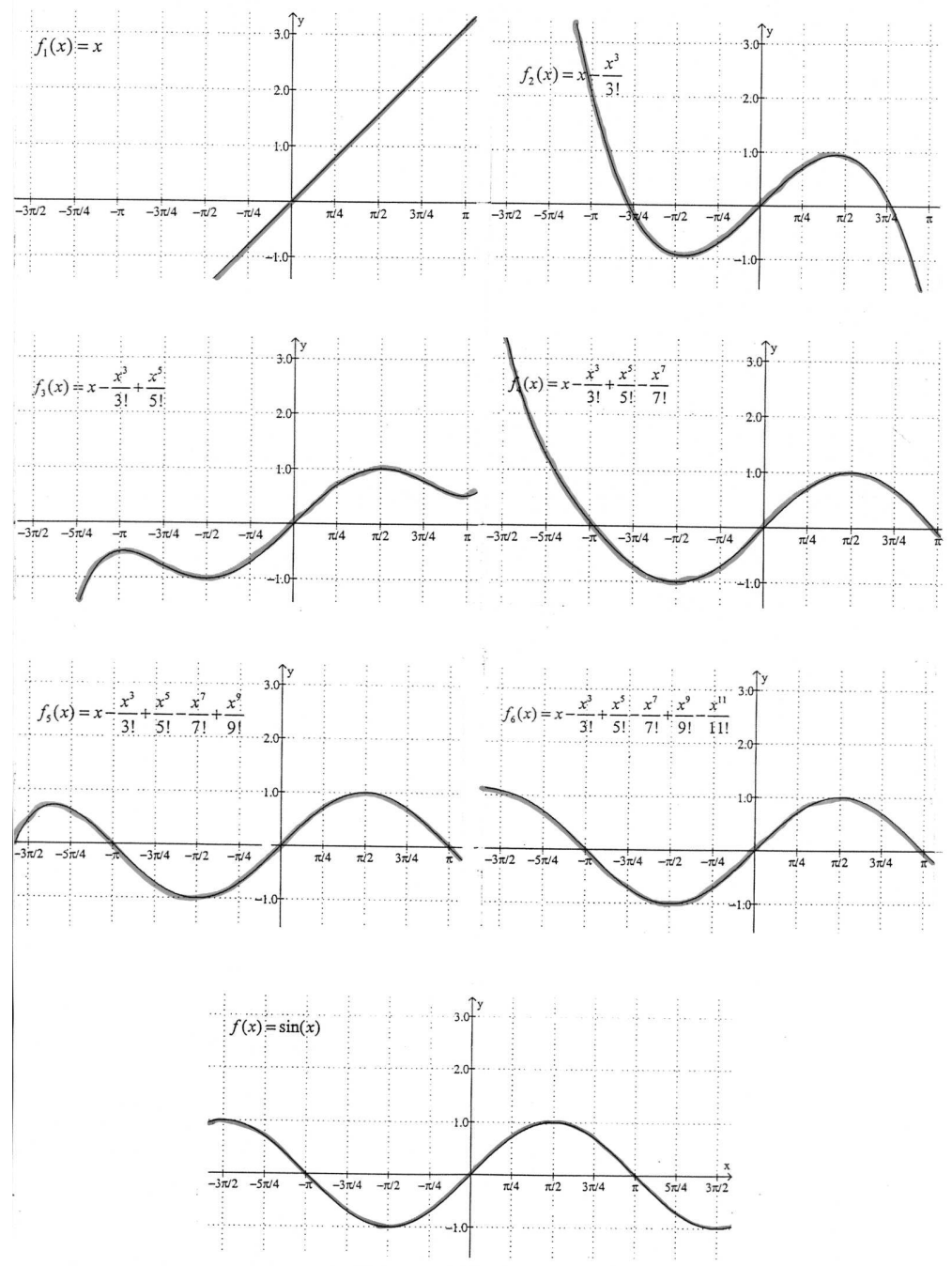

Figure 1 
But it does not mean that theoretical discussion is de-valued in any way because of that. Let us look at the case of $f(x)=1 /\left(1+x^{2}\right)$ (see Figure 2).
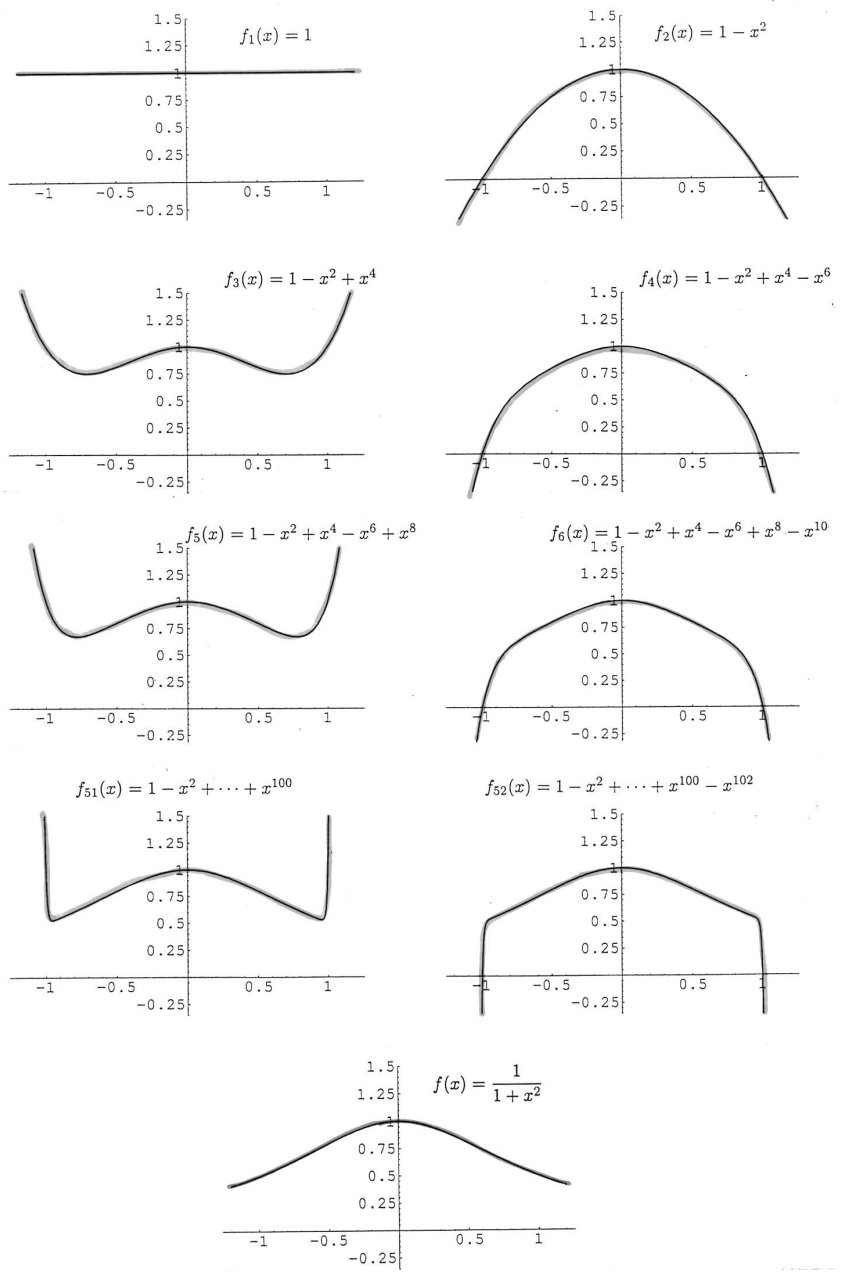

Figure 2

What is the difference between the situation of this function and the previous sine function? Computing more and more terms would not yield any clue. Students will get perplexed, which is a good thing. What happens outside the open interval $(-1,1)$ in the case of $f(x)=1 /\left(1+x^{2}\right)$ ? Plugging in 1 or -1 for $x$ in $f(x)$ still yields no clue. The full explanation only becomes transparent when the problem is looked at from a theoretical standpoint, as a complex-valued function on $\mathbb{C}$.

If polynomial functions cannot do the job, what other type of functions can be used? A mixture of theory plus computer illustration will convince students of the power of Fourier series (see Figure 3). 


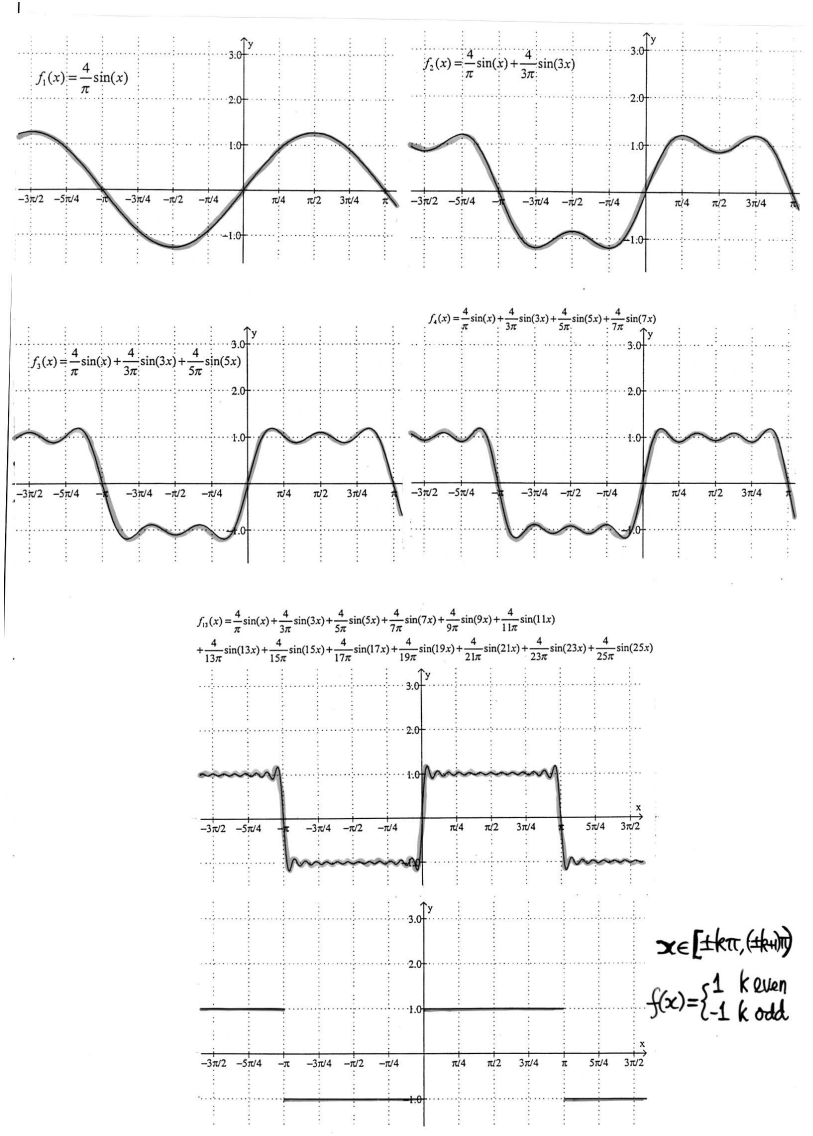

Figure 3

(2) There is a well-known theorem in plane euclidean geometry obtained by Pierre Varignon in the 17th century: If $A, B, C, D$ are the midpoints of the four sides of a quadrilateral $P Q R S$, then $A B C D$ is a parallelogram (See Figure 4).

Theorem If $A, B, C, D$ are the midpoints of the sides of a quadrilateral $P Q R S$, then $A B C D$ is a parallelogram.

(Pierre Varignon, $17^{\text {th }}$ century)

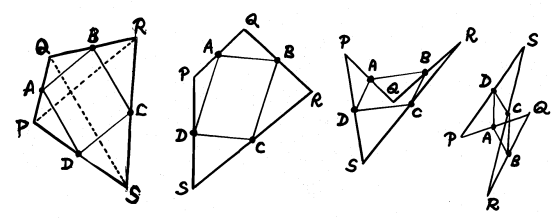

Figure 4

Using the computer software CABRI or SKETCHPAD the students can vary the shape of $P Q R S$ and discover, to their amazement, that the midpoints $A, B, C, D$ always form a parallelogram. Then they can start to think about how to prove (explain) this interesting phenomenon. 
Celia Hoyles of the Institute of Education at University of London offers a deeper observation, namely, a strong converse of Varignon's Theorem [4]. Start with four given points $A, B, C, D$. Pick any point $P$ to begin the following construction. Join $P A$ and produce to $Q$ such that $P A=A Q$. Join $Q B$ and produce to $R$ such that $Q B=B R$. Join $R C$ and produce to $S$ such that $R C=C S$. Join $S D$ and produce to $T$ such that $S D=D T$. In general one does not expect that $T$ and $P$ coincide. If $T$ and $P$ coincide, then we have a quadrilateral $P Q R S$ with $A, B, C, D$ as the midpoints of the four sides. Hence, it is interesting to ask when $T$ and $P$ coincide. Again, using CABRI or SKETCHPAD students will soon discover that as $P$ varies, $T P$ is a line segment of constant length pointing in a fixed direction. This will yield a clue to a proof (using vectors) that $T$ and $P$ coincide if and only if $A B C D$ is a parallelogram.

How I wish I had CABRI or SKETCHPAD in my school days! I love working on problems in plane euclidean geometry. Looking back, in school geometry I tasted the joy of discovery and the joy of succeeding in understanding something which was tangible (you can at least draw some pictures even if you do not know why it has to be like that at first) but not obvious (you do not know why it is like that at first). Geometry is a subject in which one can exercise logical discipline and free imagination at the same time [15]. In my school days I drew a lot of pictures in trying to get familiar with a problem and to understand more about it. But no matter how many pictures I draw the effort is no comparison in effectiveness and illumination to the use of CABRI and SKETCHPAD.

(3) The final example is also on plane euclidean geometry but tries to convey a different message. It is taken from the work of Francis Lopez-Real and Allen Leung of the Faculty of Education at University of Hong Kong [7]. (Their work covers a much more extensive scope on DGE (Dynamic Geometry Environment).) They first ask students to use CABRI to solve the problem: Draw any line segment $A B$ and devise a construction that will trisect $A B$. The construction is quite simple. Take an arbitrary point $C$ on $A B$ and draw a circle with centre at $C$ and with radius equal to $A C$, cutting $A B$ at $D$. Then draw a circle of the same size with centre at $D$, cutting $A B$ at $P$. Now, drag $C$ along $A B$ until $P$ coincides with $B$. At this point, $C$ becomes $C^{\prime}$ and $D$ becomes $D^{\prime}$, and $A C^{\prime}=C^{\prime} D^{\prime}=D^{\prime} B$.

The fun starts when one tries to apply a similar idea to a given angle $\angle A O B$. Take an arbitrary point $C$ on the circular arc $A B$ and draw equal angles $\angle A O C, \angle C O D$, $\angle D O P$ with $D, P$ on the circular arc $A B$. Now, drag $C$ along the circular arc $A B$ until $P$ coincides with $B$. At this point, $C$ becomes $C^{\prime}$ and $D$ becomes $D^{\prime}$, and $\angle A O C^{\prime}=$ $\angle C^{\prime} O D^{\prime}=\angle D^{\prime} O B$ (see Figure 5). 
Q. Draw any line segment $\mathrm{AB}$. Devise a construction that will trisect $\mathrm{AB}$.

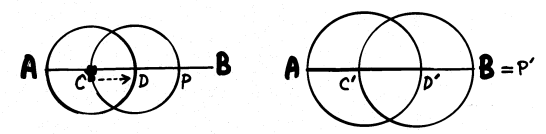

Q. Draw any angle $\angle$ AOB. Devise a construction that will trisect $\angle \mathrm{AOB}$.
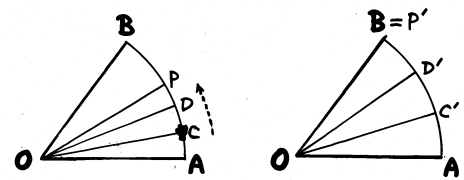

Figure 5

Is there any essential difference between the two constructions?

From a theoretical standpoint, we know that classical geometric construction can handle the trisection of a segment but not the trisection of an angle. Hence, there must have been something extra thrown into this DGE. It would be interesting to investigate what kind of construction problems can be accomplished in this new environment, just as mathematicians of the past settled the analogous question in the classical euclidean environment. It also reminds me of the influence of technology on the development of theoretical mathematics. Just as the straightedge and compasses stimulated the ancient Greeks to study construction problems that led to so much ramifications and new mathematics [8], it would not be at all surprising to envisage that IT will lead to new mathematics in years to comes.

\section{Epilogue}

In January of 2004 The Copenhagen Post carried a headline that read:

"Picking up the pieces: Lego posts an historic loss for 2003, and announces a management shakeup."

Lego, the Danish giant toy company experienced a deficit of 188 million Euros in 2003. (The name of the company comes from the Danish words $L E g$ GOdt, meaning "play well.") One reason for the downturn is seen to be the tactic of investing too heavily in recent years on products tied to films or books! Such 'target-oriented' products are as 'one-off' as they are elaborate and fashionable. In contrast, a set of simple Lego bricks can be combined in numerous ways depending on the creativity of the player. Hence, Kjeld Kirk Kristiansen, 
the CEO of the Lego Group, announced that the company is going to return to their core product, the Lego brick.

What lesson can a mathematics teacher learn from this episode? I would see it as a strong reminder that, instead of making extensive use of IT in the classroom for specific usage, it proves more profitable in the long run to concentrate on the basics. What are the basics that students need to learn and to master? How should IT be employed to enable our students learn better but not to limit their abilities to think critically and in depth? How can we ensure that a discovery approach is not to be equated with a hit-and-miss tactic, that an imaginative attitude is not to be equated with a cavalier attitude, that multi-tasking needs not be identified as sloppy and hasty work, and that the use of IT is not to be identified as following instructions (step by step) without thinking? These would be questions for a mathematics educator in this IT age to spend time on.

Coming back to classical music, which is being referred to in Section 2, let me share with readers an excerpt of the essay "Out of tune" that appeared on April 5, 2003 in Financial Times:

"... Bred and nurtured successively by church, aristocracy and bourgeoisie, classical music was ill-equipped to survive in a microwave culture. Its values are those of discipline, concentration, self-improvement, individualism, spiritual/philosophical contemplation - the values of an educated minority ..."

Again one can substitute the word "mathematics" for the word "classical music" in the excerpt. A similar, even stronger, sentiment is expressed by Julian Johnson in [5, p.89]:

"We live in a digest culture in which an unwillingness to engage in sustained thought rapidly becomes a hostility toward it. Before long, the hostility masks an incapacity to do so."

The danger of avoidance of sustained thought is quite real. We have to recognize that there exists an internal 'incompatibility' of the salient features of the subject mathematics and the surrounding culture in this IT age. However, this 'incompatibility' needs not become a 'contradiction'. We have to recognize that there is a tension between distraction and contemplation in this IT age. However, we cannot afford to be on the move all the time without ever pausing to sit down and think hard. To conclude let me quote from the very ancient Chinese Classic Da Xue (The Great Learning):

"The point where to rest being known, the object of pursuit is then determined; and, that being determined, a calm unperturbedness may be attained to. To that calmness there will succeed a tranquil repose. In that repose there may be careful deliberation, and that deliberation will be followed by the attainment of the desired end. Things 
have their root and their branches. Affairs have their end and their beginning. To know what is first and what is last will lead near to what is taught in the Great Learning." (translation in [6, pp.356-357])."

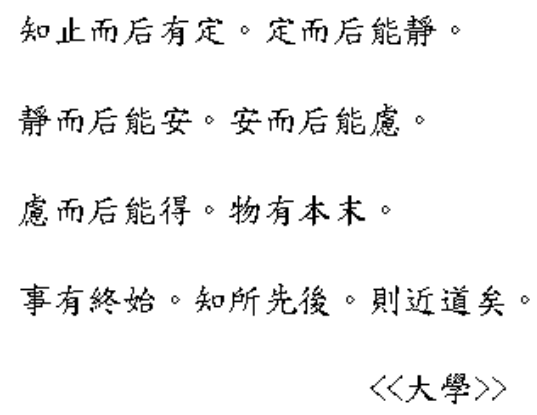

\section{References}

[1] Bardini, T., Bootstrapping: Douglas Engelbart, Coevolution, and the Origins of Personal Computing, Stanford University Press, Stanford, 2000.

[2] Engelbart, D.C., A conceptual framework for the augmentation of man's intellect, in Computer-supported Cooperative Work: A Book of Readings, edited by I. Greif, Morgan Kaufmann Publishers, San Mateo, 1988, 35-65; originally published in Vistas in Information Handling, Volume I, edited by P. Howerton, Spartan Books, Washington, D.C., 1963, 1-29.

[3] Feller, W., The problem of $n$ liars and Markov chains, Amer. Math. Monthly, 58 (1951), 606-608.

[4] Hoyles, C., Varignon's big sister, in The Changing Shape of Geometry: Celebrating a Century of Geometry Teaching, edited by C. Pritchard, Cambridge University Press, Cambridge, 2003, 177-178.

[5] Johnson, J., Who Needs Classical Music? Cultural Choice and Musical Value, Oxford University Press, Oxford, 2002.

[6] Legge, J., The Chinese Classics, Volume I: Confucian Analects, the Great Learning, the Doctrine of the Mean, Clarendon Press, Oxford, 1893; reprinted edition, Hong Kong University Press, Hong Kong, 1960.

[7] Lopez-Real, F., Leung, A.Y.L., Dragging as a conceptual tool in dynamic geometry environments, to appear in International Journal of Mathematics Education in Science and Technology.

[8] Martin, G.E., Geometric Constructions, Springer-Verlag, New York, 1998.

[9] Mason, J.H., Mathematics Teaching Practice: Guide for University and College Lecturers, Horwood Publishing, Chichester, 2002.

[10] Negroponte, N., Being Digital, Coronet Books, London, 1996. 
[11] Papert, S., The Children's Machine: Rethinking School in the Age of the Computer, Basic Books, New York, 1993.

[12] Papert, S., The Connected Family: Bridging the Digital Generation Gap, Longstreet Press, Athens, 1996.

[13] Poincaré, H., Les définitions en mathématiques, L'Enseignement Mathématique, 6 (1904), 255-283 (English translation in Science and Method by H. Poincaré, 1914; reprinted in 1952, 1996).

[14] Siu, M.K., "Less is more" or "less is less"? Undergraduate mathematics education in the era of mass education, Themes in Education, 1:2 (2000), 163-171.

[15] Siu, M.K., Learning and teaching of analysis in the mid twentieth century: a semipersonal observation, in One Hundred Years of L'Enseignement Mathématique: Moments of Mathematics Education in the Twentieth Century, edited by D. Coray et al, L'Enseignement Mathématique, Genève, 2003, 179-190.

[16] Stoll, C., Silicon Snake Oil: Second Thoughts on the Information Highway, Anchor Books, New York, 1996.

[17] Stoll, C., High-Tech Heretic: Why Computers Don't Belong in the Classroom and other Reflections by a Computer Contrarian, Doubleday, New York, 1999. 